\title{
Complexity in simplicity: monogenic disorders and complex cardiomyopathies
}

\author{
Ju Chen ${ }^{1}$ and Kenneth R. Chien ${ }^{1,2}$ \\ ${ }^{1}$ University of California-San Diego Salk NHLBI Program in Molecular Medicine, Department of Medicine and \\ ${ }^{2}$ Center for Molecular Genetics, University of California-San Diego School of Medicine, La Jolla, California 92093, USA
}

Address correspondence to: Kenneth R. Chien. Tel: (619) 534-6835, Fax: (619) 534-8081, E-mail: kchien@ucsd.edu.

"For every complex buman problem there is a neat and simple answer that is wrong ..." - H.L. Mencken

Unraveling complex human diseases has never been a simple task. A prime example of the challenge of complex diseases is offered by reflecting on the breathtaking advances in our understanding of the pathways that lead to human cardiomyopathies. Cardiomyopathies have traditionally been considered to represent diseases that primarily affect cardiac muscle, although in many cases cardiac dysfunction arises due to a secondary effect on the heart from an initial insult outside of cardiac muscle per se. As such, the pathways that drive the onset and progression of the disease often reflect the combined effects of genetic susceptibility, environmental stimuli, and hormonal and physiological cues from other organ systems.

Caridomyopathies can be inherited or acquired, and they are classified into 3 phenotypic classes: hypertrophic cardiomyopathy (HCM), dilated cardiomyopathy (DCM), and restrictive cardiomyopathy (RCM). Each class has distinct morphological, physiological, and clinical endpoints, which may reflect discrete signaling pathways. In support of this concept, a growing body of evidence indicates that several in vivo phenotypic features of HCM, DCM, and RCM can be distinguished at the single-cell level (1). The challenge has been to identify the pathways that drive these single cell phenotypes and to design a biologically targeted approach to interrupt or suppress critical signals required for the pathological response. In this regard, a combination of insights from human genetics, molecular and cellular biology, and genetically engineered animal models is beginning to make progress toward this long-term goal.

Since the Seidman's initial discovery of mutations in the $\beta$-myosin heavy chain gene (2), a growing number of mutations have been found in compo- nents of the primary cardiac cytoarchitecture, including components of the cardiac contractile sarcomere, and the intrasarcomeric, and extrasarcomeric cytoskeleton $(2,3,7)$. The cytosol of all eukaryotic cells contains a network of protein filaments and associated proteins which collectively constitute the cytoskeleton. Among these filaments are the actin microfilaments, the somewhat wider tubulin microtubules, and the intermediate filaments. To perform force-generating contraction, and to accommodate the repetitive changes in cell geometry that occur during each cardiac cycle, cardiac myocytes have evolved an abundant and highly specialized cytoskeleton, whose components can be classified into the following 3 groups:

(a) The force-generating contractile sarcomeric cytoskeleton consists of a highly-ordered arrangement of myosin thick filaments, actin thin filaments, and associated proteins, such as the troponin-tropomyosin complex. The repeating sarcomere units are arranged in series, resulting in striated myofibrils.

(b) The intrasarcomeric cytoskeleton contains titin, $\alpha$-actinin, myosin binding protein $\mathrm{C}$, and other proteins that anchor myofilaments and their sarcomeric units and regulate the displacement of myofilaments during each contraction cycle.

(c) The extrasarcomeric cytoskeleton is composed of desmin and lamin-containing intermediate filaments that provide a link between adjacent myofibrils and a framework for the nuclear envelope. This extrasarcomeric network also includes subsarcolemmal proteins that provide a connection between the peripheral myofibrils to the sarcolemma and to the extracellular matrix. These include the subsarcolemmal proteins dystrophin and its associated glycoproteins which link the sarcomere to the extracellular matrix through laminin (Figure 1). The extrasarcomeric cytoskeleton also contains a complex consisting of talin, and vinculin (metavinculin) which binds integrins, thereby connecting with the extracellular matrix (Figure 1).

Recent studies of monogenic forms of hypertrophic cardiomyopathies have provided an initial insight into the importance of this cytoskeleton-sarcomeric network for the maintenance of normal cardiac structure and function. HCM, a disorder characterized by left and/or right ventricular hypertrophy, may be asymmetric and often involves the interventricular septum. In the majority of cases, HCM is inherited. Seven different genes - $\beta$-myosin heavy chain, ventricular myosin light chain 1 , ventricular myosin light chain 2 , cardiac troponin $\mathrm{T}$, cardiac troponin I, a tropomyosin, and myosin binding protein C - have been found to cause familial HCM in humans $(2,3)$. These 7 genes encode protein components of the contractile sarcomere itself $(\beta$-myosin heavy chain, myosin light chain 1 and 2, troponin $\mathrm{T}$ and $\mathrm{I}, \alpha$-tropomyosin) or of the intrasarcomeric cytoskeleton (myosin binding protein $\mathrm{C}$ ). While the pathway by which these mutations lead to HCM remains unclear, research suggests that secondary activation of Ras and other $G$ protein signaling pathways may account for the phenotype (1).

In contrast to HCM, DCM is characterized by ventricular chamber dilation and wall thinning, which is accompanied by severe systolic contractile dysfunction. Inherited gene defects account for up to $25 \%$ of the cases of DCM, with toxic, metabolic, infectious agents or other environmental factors accounting for the remaining cases. One gene identified for DCM is a component of the extrasarcomeric cytoskeleton, dystrophin $(4,5)$. In this regard, it is of interest to note that Coxsackie virus B3 


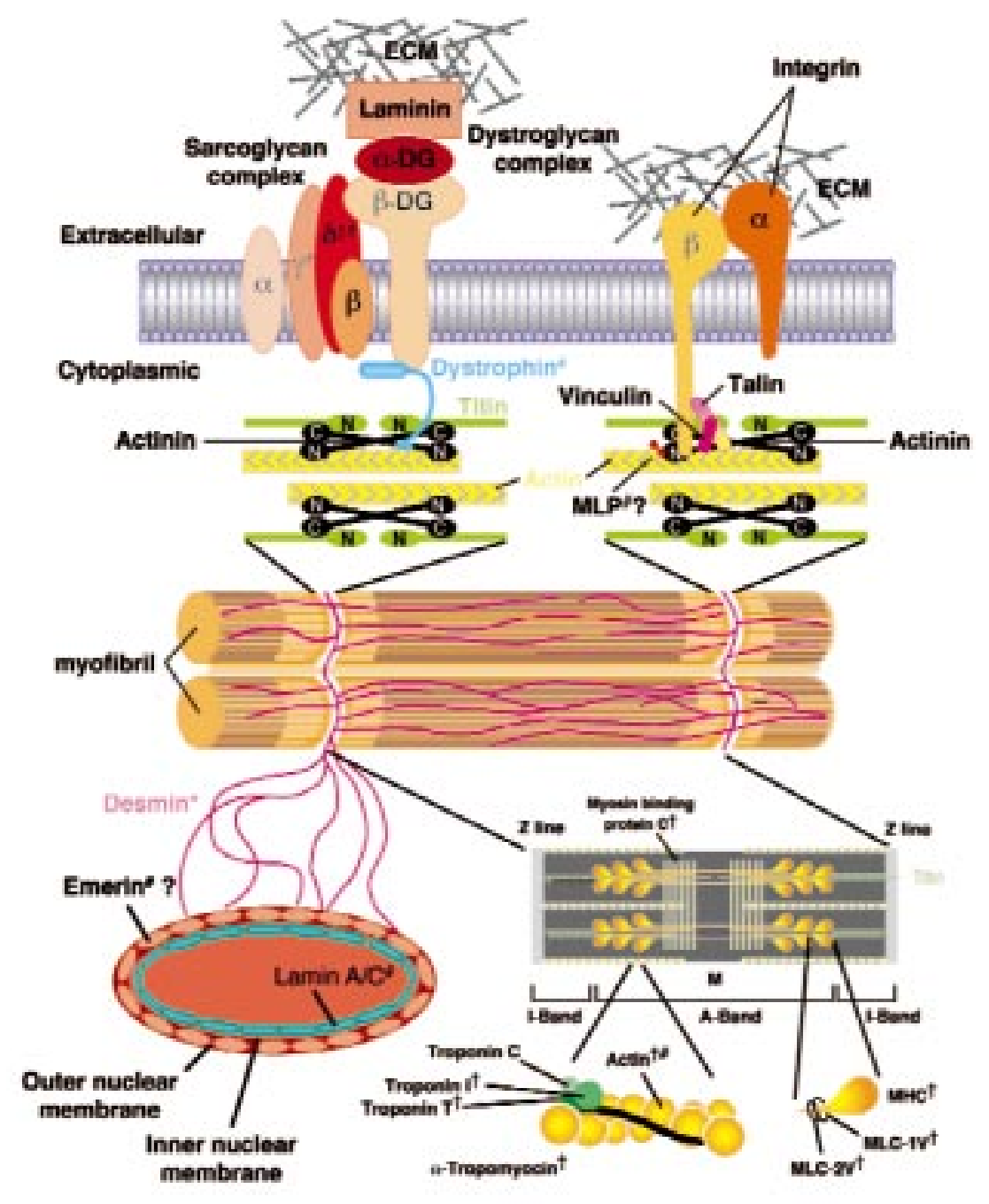

Figure 1

The cytoskeletal protein network in cardomyocytes. To simplify the figure, only selective cytoskeletal proteins have been shown. "?" indicates that the location of the protein is uncertain. Mutations in genes that can cause HCM, DCM, and RCM are indicated by $\dagger$, \#, and *, respectively. Note that mutations in both $\alpha$-cardiac actin (human) and $\delta$-sarcoglycan (hamster) can result in either HCM or DCM.

can cause DCM and cleaves dystrophin, thereby impairing the function of dystrophin and its associated glycoproteins (6). Mutations in the intermediate filament protein, nuclear lamin (lamin $\mathrm{A} / \mathrm{C}$ ), and a novel nuclear membraneassociated protein, emerin, can result in Emery-Dreifuss muscular dystrophy that can be associated with DCM (3). Another disease gene identified for DCM is $\alpha$-cardiac actin (7), which has been regarded as a component of the sarcomere, although more recent evidence suggests it may play an important role in the intrasarcomeric cytoskeleton as well (see further discussion below). In cytoskeletal proteins which disrupt cell membrane stability and/or impair force transmission will be manifest as DCM. Although evidence that mutations of a single cytoskeletal protein can give rise to either DCM or HCM would appear to contradict this paradigm, further analysis of the specific mutations within $\alpha$-cardiac actin which result in DCM or HCM allow for an interpretation consistent with this paradigm, as discussed by Mogensen et al.

Missense mutations of cardiac actin which cause DCM are located in the immobilized end of $\alpha$-actin which is thought to mediate cross binding of anchor proteins to the $Z$ lines within the sarcomere (7). Therefore, this portion of $\alpha$-cardiac actin would function to mediate force transmission of cardiomyocytes and/or to be required for membrane stability. In contrast, Mogensen et al. have found a mutation in the potential myosin binding region of $\alpha$-cardiac actin in association with HCM. This portion of the protein most likely would be directly involved in sarcomeric contraction.

It should be noted, however, that there are alternative explanations why mutations in $\alpha$-cardiac actin can result in either DCM or HCM. Of these, the most compelling would be based on the effects of diverse genetic backgrounds. This possibility is supported by recent results with a hamster model of cardiomyopathy. A single deletion within the $\delta$-sarcoglycan gene, encoding a protein in the dystrophin glycoprotein complex, can cause either DCM or HCM in mutant hamsters, depending on the specific genetic backgrounds (9). To understand underlying mechanisms for the divergent manifestation of specific forms of human cardiomyopathy, it will be important to generate mouse models in which specific residues within disease genes have been modified in the germ line. Breeding these mutations into distinct genetic backgrounds, and studying the influence of diverse backgrounds on functional and pathological effects of the mutations, will ultimately allow us to identify potential modifier genes.

In agreement with human genetic studies, conventional knockout or naturally occurring mutant mice which have mutations in cytoskeletal proteins such as muscle-lim-protein (MLP) (10), $\gamma$ sarcoglycan (11), and desmin $(12,13)$ also display characteristics of cardiomyopa- 
thy. However, cardiac-specific expression of a variety of different signaling molecules in transgenic mice can also result in various forms of cardiomyopathy. These signaling molecules include Goq, Ras, PKC, CREB, and calcineurin (1). More recently, a cardiac-specific knockout of gp130 in mice has revealed a role for a myocyte survival pathway as a genetic modifier that acts to inhibit the onset of DCM following biomechanical stress (14). The recent establishment of Cre-lox technology for generating ventricular cardiomyocyte restricted mutations in mice should facilitate distiguishing whether genetic pathways that promote or suppress cardiomyopathy are intrinsic or extrinsic to cardiomyocytes (15).

To date, disease genes mutated in human familial cardiomyopathies have been found to be primarily cytoskeletal proteins whose expression is relatively restricted to muscle, but this does not necessarily indicate that only cytoskeletal proteins are involved in cardiomyopathy. In this regard, it should be noted that mutations in genes encoding proteins essential for cardiac energetics can also cause DCM and HCM (16). Clearly, results from genetically engineered animal models support the concept that many other genes can contribute to the development of cardiomyopathy. However, germ line mutation in many cases result in embryonic lethality. Therefore, it may be unlikely to find mutations in these genes in familial cases of cardiomyopathy. However, allelic variation and/or aberrant regulation of these genes may play a very important role in acquired cardiomyopathy and also in the process of disease progression during familial cardiomyopathy. Rapid screening for these allelic variants in subsets of high risk patients is likely to be aided by the development of custom oligonucleotide DNA arrays.

In summary, the study of simple monogenic cardiomyopathies has revealed a level of molecular, cellular, and genetic complexity that matches the disease process itself. Fortunately, a new wave of genomic technology in animal models and humans holds great promise for resolving the next level of complexity. The impending availability of a complete set of human expression sequence tags, as well as their chromosomal locations will expedite the conventional analysis of monogenic cardiomyopathies where the loci are known, but the disease genes have yet to be identified. Insights from large-scale chemical mutagenesis screens in zebrafish and the mouse for embryonic and postnatal forms of cardiomyopathy should be forthcoming. The hope is that we may eventually find simplicity in the complexity of human cardiomyopathies.

\section{Acknowledgments}

Because of space limitations, we were unable to cite many important papers in this field. We would like to thank Sylvia Evans for constructive suggestions, and Kirk Knowlton, Paul Grossfeld, and Cornel Badorff for critical reading of this manuscript.

1. Hunter, J.J., and Chien, K.R. 1999. Signaling pathways for cardiac hypertrophy and failure. N. Engl. J. Med. In press.

2. Seidman, C.E., and Seidman, J.G. 1999. Molecular genetics of inherited cardiomyopathies. In Molecular basis of cardiovascular disease. K.R. Chien, editor. W.B Saunders. Philadelphia, PA. 251-263.
3. Bonne, G., Carrier, L., Richard, P., Hainque, B., and Schwartz, K. 1998. Familial hypertrophic cardiomyopathy: from mutations to functional defects. Circ. Res. 83:580-593.

4. Towbin, J.A. 1998. The role of cytoskeletal proteins in cardiomyopathies. Curr. Opin. Cell Biol. 10:131-139.

5. Campbell, K.P. 1995. Three muscular dystrophies: loss of cytoskeleton-extracellular matrix linkage. Cell. 80:675-679.

6. Badorff, C., et al. 1999. Enteroviral protease 2A cleaves dystrophin: evidence of cytoskeletal disruption in an acquired cardiomyopathy. Nat. Med. 5:320-326.

7. Olson, T.M., Michels, V.V., Thibodeau, S.N., Tai, Y.S., and Keating, M.T. 1998. Actin mutations in dilated cardiomyopathy, a heritable form of heart failure. Science. 280:750-752.

8. Mogensen, J., et al. $\alpha$-cardiac actin is a novel disease gene in familial hypertrophic cardiomyopathy. J. Clin. Invest. 103:R39-R43.

9. Sakamoto, A., et al. 1997. Both hypertrophic and dilated cardiomyopathies are caused by mutation of the same gene, delta-sarcoglycan, in hamster: an animal model of disrupted dystrophin-associated glycoprotein complex. Proc. Natl. Acad. Sci. USA. 94:13873-13878.

10. Arber, S., et al. 1997. MLP-deficient mice exhibit a disruption of cardiac cytoarchitectural organization, dilated cardiomyopathy, and heart failure. Cell. 88:393-403.

11. Hack, A.A., et al. 1998. Gamma-sarcoglycan deficiency leads to muscle membrane defects and apoptosis independent of dystrophin. J. Cell Biol. 142:1279-1287.

12. Li, Z., et al. 1996. Cardiovascular lesions and skeletal myopathy in mice lacking desmin. Dev. Biol. 175:362-366.

13. Milner, D.J., Weitzer, G., Tran, D., Bradley, A., and Capetanaki, Y. 1996. Disruption of muscle architecture and myocardial degeneration in mice lacking desmin. J. Cell Biol. 134:1255-1270.

14. Hirota, H., et al. 1999. Loss of a gp130 cardiac muscle cell survival pathway is a critical event in the onset of heart failure during biomechanical stress. Cell. 97:189-198.

15. Chen, J., Kubalak, S.W., and Chien, K.R. 1998. Ventricular muscle-restricted targeting of the RXRalpha gene reveals a non-cell-autonomous requirement in cardiac chamber morphogenesis. Development. 125:1943-1949.

16. Mathur, A., et al. 1999. Molecular heterogeneity in very-long-chain acyl-CoA dehydrogenase deficiency causing pediatric cardiomyopathy and sudden death. Circulation. 99:1337-1343. 